\title{
A Successful Walk-In Psychiatric Model for Integrated Care
}

\author{
David S. Kroll, MD, Carol Latham, LICSW, Janice Mahal, LICSW, \\ Meagan Siciliano, LICSW, Laura S. Shea, LICSW, Lisa Irwin, Bonnie Southworth, MD, \\ and David F. Gitlin, MD
}

Purpose: Accommodating walk-in psychiatry visits in primary care can improve access to psychiatric care for patients from historically underserved groups. We sought to determine whether a walk-in psychiatry model embedded within an integrated care practice could be sustained over time, and to characterize the patients who accessed care through it.

Methods: We reviewed electronic health records linked to 811 psychiatry encounters in an integrated care practice between October 1, 2015 and September 30, 2017. Primary outcomes were the initial and return psychiatry encounters per month. Secondary outcomes were the demographics and diagnoses of patients who accessed their initial visits through walk-in sessions and scheduled appointments.

Results: 490 initial psychiatry evaluations and 321 return encounters took place over the 2-year study period. The volume of initial psychiatry evaluations per month did not significantly change, but the volume of psychiatry follow-up encounters significantly increased after the walk-in session expanded. Medicaid recipients (OR, 1.9; 95\% CI, 1.2 to 3.0); individuals without a college degree (OR, 1.7 ; $95 \%$ CI, 1.1 to 2.5$)$; individuals who were single, divorced, or separated (OR, 1.7; 95\% CI, 1.1 to 2.5); and individuals who identified as Black or Hispanic (OR, 2.5; 95\% CI, 1.7 to 3.6) were more likely to access an initial psychiatry evaluation through a walk-in session as opposed to a scheduled appointment.

Conclusions: Providing psychiatric care on a walk-in basis in integrated care is sustainable. Patients from historically underserved groups may access psychiatric care disproportionately through a walk-in option when it is available. ( $\mathrm{J}$ Am Board Fam Med 2019;32:481-489.)

Keywords: Behavioral Medicine, Medicaid, Minority Health, Psychiatry, Primary Health Care, Underserved Populations

Missed health care appointments are common in psychiatry and disproportionately affect patients from historically underserved demographic groups. ${ }^{1,2}$

This article was externally peer reviewed.

Submitted 3 December 2018; revised 5 February 2019; accepted 20 February 2019.

From the Department of Psychiatry (DSK, LI, DFG), Division of Social Work (CL, JM, MS, LSS), and Department of Medicine (BS), Brigham and Women's Hospital, Boston, MA; Harvard Medical School, Boston, MA (DSK, BS, DFG).

Funding: The submitted work was conducted with support from Brigham and Women's Hospital via the H. Richard Nesson Fellowship.

Conflict of interest: DSK received a speaking honorarium from Avasure, LLC in 2017. All other authors have no competing interests to disclose.

Corresponding author: David S. Kroll, MD, Department of Psychiatry, Brigham Health, 75 Francis Street, Boston, MA 02115 (E-mail: dskroll@bwh.harvard.edu).
Younger age, racial minority status, Medicaid enrollment, low educational level, and living in an underserved geographic area are all associated with missing scheduled health appointments. ${ }^{3-10}$ This should not be surprising because patients with fewer social resources face more practical barriers to keeping appointments, such as housing insecurity and transportation failure, and frequently must juggle priorities that directly compete with their personal health. ${ }^{11-13}$ Missed appointments, in turn, not only disrupt care but are additionally associated with worse health outcomes, higher medical comorbidity, and higher utilization of emergency services. ${ }^{3-5,14-16}$ Thus, they contribute to a vicious cycle in which the patient groups who are already the most vulnerable to negative health outcomes may increasingly be driven to the margins by systems that do not consistently meet their needs. 
"Integrated care," a term that applies to models of delivering behavioral health services through ambulatory medical settings other than a dedicated behavioral health clinic (of which primary care is the most common), can facilitate improved behavioral health outcomes for patients even when they do not have the opportunity to meet regularly with a psychiatrist. ${ }^{17-19}$ Integrated care approaches typically apply multidisciplinary teams to address the medical and nonmedical issues that patients with common behavioral health conditions face in a variety of ways. ${ }^{20}$ When a face-to-face visit with a psychiatrist is indicated, however, most integrated care models still require a patient to schedule this appointment in advance and keep it. A notable exception may be the use of an asynchronous virtual encounter. ${ }^{21}$

Missed appointments can be predicted at the individual patient level by a patient's previous history of missing health appointments. ${ }^{22,23} \mathrm{We}$ previously demonstrated that missed initial psychiatry appointments can be proactively managed in integrated care by inviting newly referred patients with an historic missed health appointment rate of $20 \%$ or higher to a weekly walk-in psychiatry session, and that under this model a higher volume of patients from historically underserved groups accessed psychiatric care compared with an earlier model in which all psychiatry appointments were scheduled in advance. ${ }^{22}$ Our initial report, which described the first 6 months after our model was implemented, also found a trend toward serving higher proportions of patients with Medicaid insurance, nonwhite race, and no college degree through the walk-in model, but this finding was not statistically significant. $^{22}$

Our practice subsequently doubled the time available for walk-in psychiatry visits to 2 clinical sessions per week, over 2 years, to accommodate a higher volume and variety of new referrals as well as returns, which had been excluded from the original model. We hypothesized that expanding the time for walk-in visits would be feasible, and that serving a higher volume of patients through the new clinic model over time would increase our statistical power sufficiently to detect differences in the demographics of the patients who accessed care through a walk-in session. Specifically, our team's goal was to improve access to psychiatric care for Medicaid recipients, which aligned with our hospital system's priorities, and we expected that patients with Medicaid insurance would disproportionately access psychiatric care through walk-in sessions as opposed to scheduled appointments.

\section{Materials and Methods Setting and Patients}

Our integrated care clinic is embedded within a large, hospital-based primary care practice that treats approximately 16,700 patients with a diverse array of medical comorbidities and psychosocial needs, approximately $20 \%$ of whom are on Medicaid. The primary care practice was staffed by 108 primary care providers (PCPs), including 64 residents in internal medicine, and a behavioral health team consisting of a 0.3 full-time equivalent (FTE) psychiatrist and 4 full-time clinical social workers (SWs). The clinic also employs nonphysician staff to assist with phone calls, medication refills, and prior authorizations, which allows clinicians to care for a higher volume of patients than they otherwise could.

The psychiatrist held 3 half-day clinical sessions per week. In the original model, 2 of these sessions were dedicated to scheduled visits ( 3 initial evaluations and 2 return visits), and the third was a walk-in session to which any patient with an established PCP in the clinic could access an initial psychiatry evaluation on demand. This model has been described previously ${ }^{22}$ and was designed to focus on diagnostic assessments, treatment planning, and treatment initiation rather than true longitudinal care, although many patients remained in this model longitudinally due to either their own preferences or a failure to establish longitudinal care in a traditional psychiatry clinic after referral. Notably, the original model was not designed to accommodate return visits, and all patients who requested return visits were offered scheduled appointments instead of a referral to a walk-in session. However, patients who presented to a walk-in session with a follow-up need were not turned away.

Conversion of a scheduled appointment session to a walk-in session did not involve any change in resources, personnel, or costs. Billing claims for psychiatric care provided under this model were submitted according the same contracts that other physicians in the hospital system routinely used, which were predominantly fee-for-service, regardless of whether the care was provided during a 
scheduled appointment or a walk-in encounter (ie, no special payment arrangements were negotiated for these services).

\section{Intervention}

In response to a growing number of patients requesting return visits and to a high volume of patients who continued to miss the scheduled psychiatry appointments to which they were referred, we increased our capacity for walk-in encounters in November 2016. In the new model, the psychiatrist maintained 1 half-day session for scheduled appointments and expanded the walk-in session to a full day. The social workers explicitly invited approximately 10 to 12 new patients to the walk-in psychiatry session per week, and PCPs were advised that they could refer any of their patients, for any reason, at their own discretion. Patients were invited to arrive between 9:00 AM and 3:00 PM on any Friday, to be seen as a walk-in on a first-come, first-served basis. Although they were told that checking in later than 3:00 PM could result in their not being seen, in most instances patients who checked in before 4:00 Pм still received care that day. A history of missed health appointments was no longer required for referral to a walk-in session, but patients who were referred to psychiatry and had a documented history of missing $20 \%$ or more of their health appointments were still directed preferentially to a walk-in session instead of to a scheduled appointment by the triage staff who fielded psychiatry referrals.

Most patients who received an initial psychiatry evaluation were encouraged to follow-up with their PCPs for the immediate next steps but were invited to return to the psychiatrist through either a scheduled appointment or a walk-in session if they desired additional assessment or treatment planning. Patients who appeared to require longitudinal management in a psychiatry clinic (eg, those with major psychiatric illnesses) were referred to the psychiatry clinic at the time of the assessment, but they were allowed to continue receiving integrated care (through walk-in or scheduled encounters) if this referral was unsuccessful.

\section{Measurements}

Our institution uses an electronic health record (EHR) to track and document referrals and clinical encounters. The EHR contains data on referral dates, encounter dates and times, patient demo- graphics, primary encounter diagnoses, medication orders, and billing, and we ran a query through the EHR to obtain this information for all patients who received a billed encounter with the integrated care psychiatrist during our hospital system's fiscal years (FY) 2016 and 2017 (ie, from October 2015 to September 2017). No patients who received a billed encounter with the integrated care psychiatrist were excluded from our analysis.

Patients with any private or commercial insurance carrier were coded as having "private" insurance in our data set. Some patients in our network used a hybrid insurance carrier that served both Medicaid and private insurance clients and was operated by our hospital system, called Neighborhood Health Plan. Neighborhood Health Plan clients were coded separately from Medicaid and private insurance recipients in our data set.

Patients were identified as having received an initial evaluation if the Current Procedural Terminology (CPT) code used to bill for the encounter was a 90792 or a 90791 (psychiatric diagnostic interview examination). Return visits were identified by CPT codes 99211 to 99215 (evaluation and management of established patients in the outpatient setting). Two patients had 2 separate encounters for which a 90792 code was used, and in these cases the encounters with the later dates of service were coded as return encounters. Two encounters were billed using codes 99203 (evaluation and management of a new patient in the outpatient setting); review of these encounters identified them to be return visits, and they were coded as such. We determined which patients had accessed the psychiatry encounter through a walk-in session by measuring the appointment lag time, which is the number of days between the date the encounter was scheduled and the date the encounter took place, as recorded in the EHR. For all walk-in visits, the recorded appointment lag time was 0 days, and for all scheduled visits the appointment lag time was 1 or more days. While the date the encounter was scheduled can be used as a proxy for the date of referral for patients who scheduled their appointments in advance, referrals to a walk-in session came from multiple sources and were not consistently documented. Therefore, lag time cannot be used to compare actual wait times between the 2 groups.

This study was approved by our Institutional Review Board. 
Figure 1. Initial psychiatry evaluations per month (XbarS Chart).

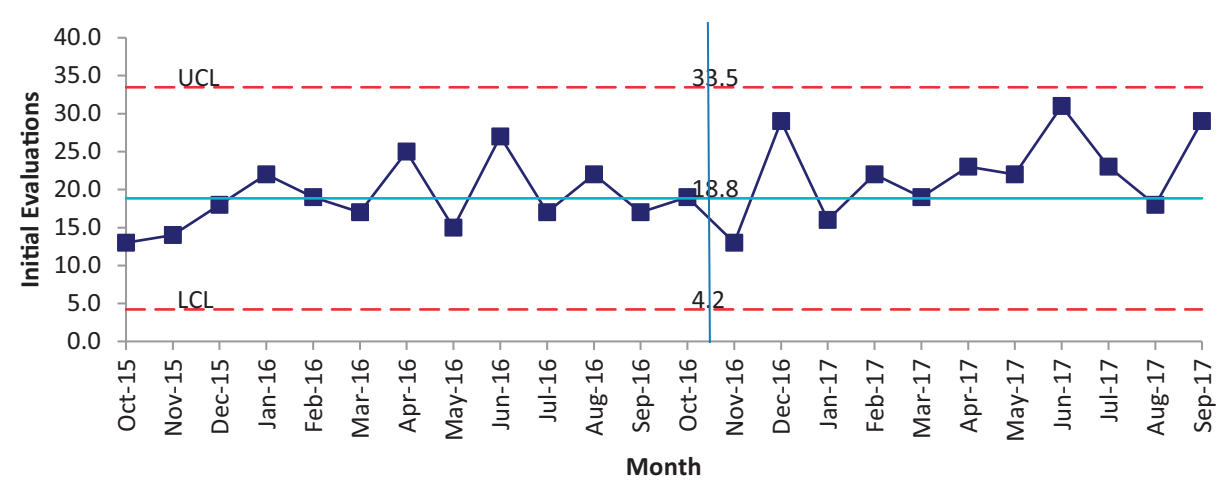

XbarS chart. UCL, upper control limit. LCL, lower control limit. Vertical line demarcates the change in the clinic structure on November 1, 2016, in which the walk-in clinic session expanded to a full day and began accommodating follow-up encounters.

\section{Statistical Analysis}

The numbers of initial and return encounters per month were plotted on statistical process control charts using the XbarS function, which is a statistical tool used to detect change within a stable system. ${ }^{24}$ Multiple rules can be used to determine whether a data point or series of data points represents routine variation or statistically significant "special cause variation." These include a single point above or below the control limits, which are marked by 2 dashed lines above and below the center line; 2 out of 3 consecutive points that are more than 2 standard deviations away from the mean; and 8 out of 9 consecutive points on the same side of the center line.

We divided the patients who received an initial encounter into 2 groups: those who accessed the first psychiatry visit through a scheduled appointment and those who accessed the first psychiatry visit through a walk-in encounter. For each group, we calculated the average age and the percentages of patients who came from each measured demographic group. A $t$ test was used to calculate a $\mathrm{p}$-value for the age variable, and statistical significance for other variables was calculated using chi squares. We calculated odds ratios (ORs) and 95\% confidence intervals (95\% CIs) for accessing the initial psychiatry visit through a walk-in encounter as opposed to a scheduled appointment for demographic and diagnostic groups where the $\chi^{2}$ test indicated statistically significant differences. We used Microsoft Excel 2016 for all calculations and QI Macros for Excel version 2015.09, by Know-
Ware International Inc., to generate the statistical process control chart.

\section{Results}

Figure 1 is an XbarS chart showing the number of patients per month who received an initial primary care psychiatry evaluation in FYs 2016 and 2017. The volume of new patient encounters remained consistent over the 2-year period and reached a final total of 490. No data points after the model changed meet criteria for special cause variation, indicating that the volume of initial evaluations did not significantly change when the second scheduled clinic session was converted to an additional walk-in session.

Figure 2 is an $\mathrm{XbarS}$ chart showing the number of patients per month who made a return psychiatry visit in FYs 2016 and 2017, which reached a final total of 321. After November 2016, when the clinic began explicitly allowing patients to use walk-in sessions for return visits, the number of return visits significantly increased. Three rules for special cause variation are met by the data points after the intervention, including a single point above the upper control limit, 2 out of 3 consecutive points more than 2 standard deviations away from the center line, and more than 8 out of 9 consecutive points above the center line.

Table 1 compares the characteristics of the patients who accessed their first psychiatry encounters through a scheduled appointment with those who accessed it through a walk-in visit during FY 
Figure 2. Return psychiatry encounters per month (XbarS Chart).

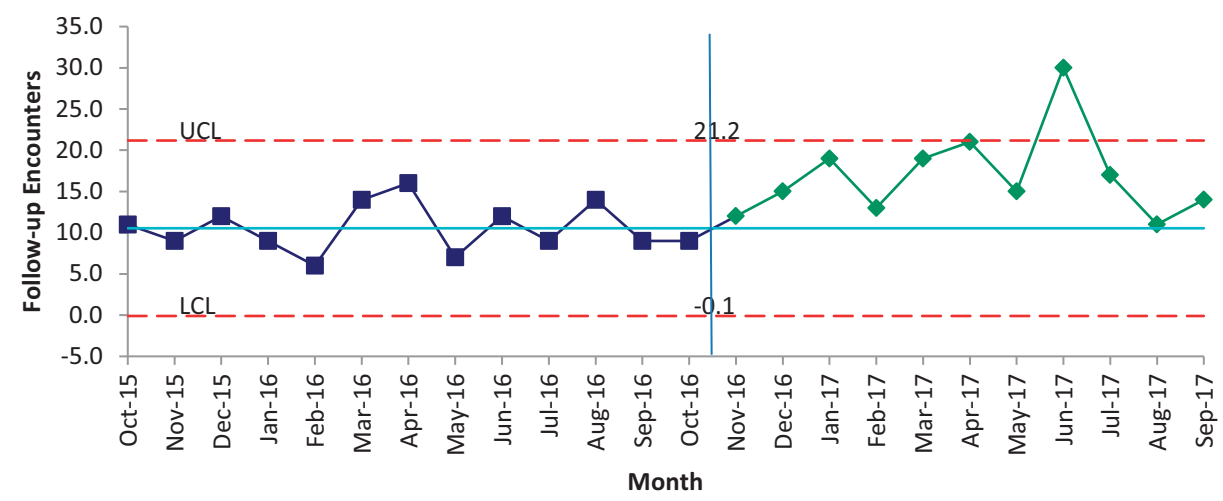

XbarS chart. UCL, upper control limit. LCL, lower control limit. Vertical line demarcates the change in the clinic structure on November 1, 2016, in which the walk-in clinic session expanded to a full day and began accommodating follow-up encounters.

2016 and 2017. Patients with Medicaid insurance (OR, 1.9; 95\% CI, 1.2 to 3.0); with a marital status of single, divorced, or separated (compared with married, partnered, or widowed) (OR, 1.7; 95\% CI, 1.1 to 2.5$)$; with no college degree (OR, 1.7; $95 \%$ CI, 1.1 to 2.5), and who identified as Black or Hispanic (compared with White/white, Asian, American Indian/Alaska Native, or Other) (OR, 2.5; $95 \%$ CI, 1.7 to 3.6 ) were significantly more likely to access care through a walk-in visit. Although the $\chi^{2}$ test also suggested statistically significant differences in diagnoses between the 2 groups, these differences were small in magnitude, and no odds ratios for accessing care through a walk-in visit based on a diagnostic group were statistically significant. A patient's likelihood of receiving a prescription for medication or of being transferred to the emergency department (ED) after the initial visit did not significantly differ based on how the visit had been scheduled.

\section{Discussion}

Providing psychiatric care to patients on a walk-in basis is a feasible and sustainable model for an integrated care practice. Implementation of the first iteration of the walk-in clinic resulted in a higher overall volume of primary care patients accessing psychiatric care, ${ }^{22}$ and this higher volume remained consistent over 2 years. Moreover, after patients were explicitly given the option to use the walk-in session for return visits, return patients accessed it more frequently. Because this more efficient model increased new and return patient volume without adding new psychiatrist time (ie, it reassigns existing psychiatrist time), supporting it has not required a funding strategy other than reimbursement through a standard fee-for-service contract. We are not able to report actual reimbursements for services, however, and cannot say whether the revenue generated by this model significantly differed from the revenue generated by the previous model, in which care was delivered exclusively through scheduled appointments to patients with a different payer mix.

Perhaps more importantly, patients from historically underserved groups, including Medicaid recipients, disproportionately accessed psychiatric care through the walk-in sessions, as opposed to through scheduled appointments. We do not know whether any patients accessed walk-in psychiatry services because they preferred this model, because this model provided faster access, or because they had previously failed to access care through a scheduled appointment. The patients who accessed walk-in psychiatry services appeared clinically similar to those who scheduled psychiatry services in advance, as indicated by their psychiatric diagnoses, their likelihood of receiving a prescription, and their rate of transfer to the ED after the initial visit. However, walk-in clinical services may be uniquely helpful to patients from historically underserved groups because they do not penalize them when 
Table 1. Characteristics of Patients Who Accessed Psychiatry for the First Time at Scheduled and Walk-In Integrated Care Encounters between October 2015 and September 2017

\begin{tabular}{|c|c|c|c|}
\hline & $\begin{array}{l}\text { No. }(\%) \\
\text { Scheduled }\end{array}$ & Walk-in & $P$-Value \\
\hline $\mathrm{N}$ & 268 & 222 & \\
\hline Age, mean (standard deviation) & $48(16)$ & $47(15)$ & .758 \\
\hline Female & $187(70)$ & $169(76)$ & .116 \\
\hline Insurance status & & & $<.0001$ \\
\hline Private & $115(43)$ & $51(23)$ & \\
\hline Medicare & $56(21)$ & $53(24)$ & \\
\hline Medicaid & $43(16)$ & $60(27)$ & \\
\hline NHP & $53(20)$ & $54(24)$ & \\
\hline None & $1(0)$ & $4(2)$ & \\
\hline Marital Status & & & .030 \\
\hline Single & $143(54)$ & $133(60)$ & \\
\hline Divorced or separated & $28(10)$ & $33(15)$ & \\
\hline Widowed & $8(3)$ & $8(4)$ & \\
\hline Married or partnered & $88(33)$ & $47(21)$ & \\
\hline Language & & & .121 \\
\hline English & $241(91)$ & $190(86)$ & \\
\hline Spanish & $19(7)$ & $28(13)$ & \\
\hline Other & $6(2)$ & $4(2)$ & \\
\hline Education & & & .037 \\
\hline Less than high school & $28(13)$ & $32(17)$ & \\
\hline High school or GED & $99(44)$ & $98(52)$ & \\
\hline College or higher & $97(43)$ & $59(31)$ & \\
\hline Race & & & $<.0001$ \\
\hline White & $127(48)$ & $62(28)$ & \\
\hline Black & $69(26)$ & $87(40)$ & \\
\hline Hispanic & $32(12)$ & $46(21)$ & \\
\hline Asian & $6(2)$ & $4(2)$ & \\
\hline Native & $0(0)$ & $1(0)$ & \\
\hline Other & $28(11)$ & $18(8)$ & \\
\hline Diagnosis & & & .034 \\
\hline Mood disorder & $144(54)$ & $115(52)$ & \\
\hline Anxiety disorder & $51(19)$ & $32(14)$ & \\
\hline Trauma-related disorder & $16(6)$ & $24(11)$ & \\
\hline Adjustment disorder & $23(9)$ & $15(7)$ & \\
\hline Attention deficit disorder & $9(3)$ & $5(2)$ & \\
\hline Psychotic disorder & $4(1)$ & $9(4)$ & \\
\hline Substance use disorder & $5(2)$ & $6(3)$ & \\
\hline $\begin{array}{l}\text { Neurological or developmental } \\
\text { disorder }\end{array}$ & $2(1)$ & $6(3)$ & \\
\hline Somatoform disorder & $2(1)$ & $2(1)$ & \\
\hline Personality disorder & $0(0)$ & $1(0)$ & \\
\hline Eating disorder & $0(0)$ & $1(0)$ & \\
\hline Other & $12(4)$ & $6(3)$ & \\
\hline
\end{tabular}

Continued

practical barriers and competing priorities disrupt their plans to attend a clinic visit. If a patient misses a planned visit to a walk-in session, he or she does not face discharge from the clinic and may attend a different session without needing to formally reschedule. 


\begin{tabular}{lcr}
\hline & $\begin{array}{c}\text { No. }(\%) \\
\text { Scheduled }\end{array}$ & Walk-in \\
\hline Prescribed medication & $187(70)$ & $167(75)$ \\
Transferred to the ED & $1(0)$ & .180 \\
\hline
\end{tabular}

ED, emergency department; GED, graduate equivalency degree; NHP, Neighborhood Health Plan.

Some demographic variables were listed as "unavailable" or "declined" in some patient records, and these were omitted from the percentage calculations and $\chi^{2}$ tests.

Of a total of 490 patient records (268 scheduled, 222 walk-in), 490 included gender, 490 included insurance status, 488 (267 scheduled, 221 walk-in) included marital status, 488 (266 scheduled, 222 walk-in) included language, 413 ( 224 scheduled, 189 walk-in) included educational level, 480 (262 scheduled, 218 walk-in) included race, and 490 (268 scheduled, 222 walk-in) included a billing diagnosis. "Other" billing diagnoses include sleep disorders, sexual disorders, learning disorders, medical diagnoses, and unspecified mental conditions. "Prescribed medication" and "Transferred to the ED" descriptors apply to the initial visit only.

Statistical significance determined by $P<.05$.

We did not officially keep track of referral sources or waiting room time in this study. Anecdotally, referral sources to the walk-in clinic were variable. Many patients were referred by their primary care clinicians or social workers; but some were referred by clinicians in the ED, others learned about it through family members or work colleagues; and still others were referred multiple times by multiple sources. Initial encounters typically lasted 40 minutes (although individual encounters might be longer or shorter, depending on need and complexity), and return encounters lasted 15 to 20 minutes. Waiting room time varied considerably from session to session and could be as long as 4 hours, although 30 to 90 minutes was a more typical range. Waiting room time commonly peaked between 11:00 $\mathrm{AM}$ and 12:00 PM, and when the wait was prolonged the medical assistantadvised patients who checked in at these times to return in the afternoon after getting lunch (without losing their place in line). Waiting room time was usually shorter in the afternoons compared with the mornings.

Although we did not directly measure staff or patient satisfaction, the behavioral health team members unanimously agreed that the walk-in sessions were more satisfying to offer to our clinic population than the scheduled sessions, and the nonsystematic feedback we received from patients and referring providers about the availability of walk-in visits was also overwhelmingly positive. Although this nonsystematically collected information cannot be used to assess true patient and provider satisfaction, it was interpreted by the behavioral health team and by administrators in primary care and psychiatry as an indication of wide support for the model. Thus, when the psychiatrist time dedicated to integrated care was reduced from 0.3 FTE to 0.2 FTE (due to the receipt of a research grant) after the end of the study period, the behavioral health team elected to abandon the remaining scheduled appointment session, which had been experienced as increasingly low-yield, and dedicate all the remaining time to walk-ins.

We are not the first group to offer psychiatric care to patients on a walk-in basis, ${ }^{25-27}$ but ambulatory care models that deliberately seek to accommodate unscheduled patients have not gained wide traction in the field of psychiatry. ${ }^{1}$ Models that facilitate same-day access to nonphysician behavioral health clinicians in primary care have historically been adopted more successfully, particularly among organizations that do not rely heavily on fee-for-service contracts for reimbursement including Kaiser-Permanente, the United States Air Force, Veterans' Affairs, and Cherokee Health Systems. ${ }^{28-30}$ However, in the absence of physician billing, such models may be difficult to launch and sustain in hospital systems that continue to use fee-for-service contracts for the time being. ${ }^{30}$ Meanwhile, providing rapid access to a psychiatrist is critical for patients who require psychopharmacologic expertise.

Thus there remains a gap in many health care systems, in which individuals with behavioral health conditions who need rapid access to psychiatric care are not able to get it through traditional ambulatory care pathways. ${ }^{31}$ Because patients who require unscheduled care often access it in emergency settings, ${ }^{1,31}$ a model that provides unscheduled psychiatric care in an ambulatory setting may also provide relief to crowded EDs, ${ }^{32}$ where pro- 
viders often feel unprepared to manage the high volume of psychiatric illness that they are expected to see. ${ }^{33,34}$ It is therefore possible that offering walk-in psychiatry services on a larger scale could yield benefits across the health care system.

The feasibility of accommodating walk-ins in primary care may not extend to a traditional psychiatry clinic. In our hospital system's model, initial evaluations in the psychiatry clinic are expected to lead to more follow-up psychiatry encounters than initial evaluations in primary care would, and therefore it would be more difficult for a clinician in the psychiatry clinic to accommodate a high volume of initial evaluations. Some psychiatry clinics also do not have ancillary staff to support the nonclinical needs, such as prior authorization for medications, that a high volume of patients is likely to have.

\section{Limitations}

This study was not a randomized clinical trial. The fact that a higher volume of Medicaid recipients accessed psychiatric care through a walk-in session does not necessarily signify that they preferred or deliberately would have chosen this route. Because Medicaid recipients are more likely to miss health appointments in general, they were also more likely to be referred preferentially to a walk-in session, which could confound our data. Because our behavioral health team relinquished control over referrals to the walk-in sessions by inviting any provider to refer any patient for any reason, we could not track how many patients were referred to a walk-in session, by whom, or for which reasons. Anecdotally, we found that many patients were referred multiple times and by multiple individuals within our system, and others who had been referred to a scheduled appointment accessed care through a walk-in session, and vice versa. Therefore, we did not think that trying to track the origin of each referral would yield interpretable data. We also did not survey patients directly about their experiences with the referral process or about their preferences for a walk-in versus a scheduled encounter.

The patients who received care in our practice disproportionately had health insurance. In areas where Medicaid has not been expanded, this model may require a funding source other than fee-forservice reimbursement. Therefore, we do not yet know how generalizable our findings are to other health care systems with different resources and populations. We do not yet know whether health outcomes after a walk-in encounter differ from outcomes after a scheduled appointment.

\section{Conclusions}

Providing walk-in psychiatry services in integrated care is a feasible and sustainable. Walk-in ambulatory services may be a critical point of access to outpatient psychiatric care for primary care patients from historically underserved groups, including Medicaid recipients, patients with low educational levels, and racial minorities.

To see this article online, please go to: http://jabfm.org/content/ 32/4/481.full.

\section{References}

1. Runnels P. Case studies in public-sector leadership: Addressing the problem of appointment nonadherence with a plan for a walk-in clinic. Psychiatr Serv 2013;64:404-406.

2. Zivin K, Pfeiffer PN, McCammon RJ, et al. "Noshows": Who fails to follow up with initial behavioral health treatment? Am J Manag Care 2009;15:105112.

3. Schectman JM, Schorling JB, Voss JD. Appointment adherence and disparities in outcomes among patients with diabetes. J Gen Intern Med 2008;23: 1685-1687.

4. Nguyen DL, Dejesus RS, Wieland ML. Missed appointments in resident continuity clinic: patient characteristics and health care outcomes. J Grad Med Educ 2011;3:350-355.

5. DuMontier C, Rindfleisch K, Pruszynski J, et al. A multi-method intervention to reduce no-shows in an urban residency clinic. Fam Med 2013;45:634-641.

6. Kaplan-Lewis E, Percac-Lima S. No-show to primary care appointments: Why patients do not come. J Prim Care Community Health 2013;4:251-255.

7. Neal RD, Lawlor DA, Allgar V, Colledge M, Ali S, Hassey A, et al. Missed appointments in general practice: retrospective data analysis from four practices. Br J Gen Pract 2001;51:830-832.

8. Shimotsu S, Roehrl A, McCarty M, et al. Increased likelihood of missed appointments ("no shows") for racial/ethnic minorities in a safety net health system. J Prim Care Community Health 2016;7:38-40.

9. Nancarrow S, Bradbury J, Avila C. Factors associated with non-attendance in a general practice super clinic population in regional Australia: A retrospective cohort study. Australas Med J 2014;7:323-333.

10. Samuels RC, Ward VL, Melvin P, et al. Missed appointments: Factors contributing to high no-show rates in an urban pediatrics primary care clinic. Clin Pediatr (Phila) 2015;54:976-982. 
11. Pieh-Holder KL, Callahan C, Young P. Qualitative needs assessment: healthcare experiences of underserved populations in Montgomery County, Virginia, USA. Rural Remote Health 2012;12:1816.

12. Ahmed SM, Lemkau JP, Nealeigh N, et al. Barriers to healthcare access in a non-elderly urban poor American population. Health Soc Care Community 2001;9:445-453.

13. Danis M, Kotwani N, Garrett J, et al. Priorities of low-income urban residents for interventions to address the socio-economic determinants of health. J Health Care Poor Underserved 2010;21:13181339.

14. Mackin RS, Areán PA. Cognitive and psychiatric predictors of medical treatment adherence among older adults in primary care clinics. Int $\mathrm{J}$ Geriatr Psychiatry 2007;22:55-60.

15. Moscrop A, Siskind D, Stevens R. Mental health of young adult patients who do not attend appointments in primary care: A retrospective cohort study. Fam Pract 2012;29:24-29.

16. Nguyen DL, Dejesus RS. Increased frequency of no-shows in residents' primary care clinic is associated with more visits to the emergency department. J Prim Care Community Health 2010;1:8-11.

17. Joseph R, Kester R, O'Brien C, Huang H. The evolving practice of psychiatry in the era of integrated care. Psychosomatics 2017;58:466-473.

18. Uga A, Kulkarni S, Heeramun V, et al. Evaluation of a model of integrated care for patients with chronic medical and psychiatric illness. Psychosomatics 2017;58:437-440.

19. Unützer J, Katon W, Callahan CM, et al. Collaborative care management of late-life depression in the primary care setting: A randomized controlled trial. JAMA 2002;288:2836-2845.

20. Dobbins MI, Thomas SA, Melton SLS, et al. Integrated care and the evolution of the multidisciplinary team. Prim Care 2016;43:177-190.

21. Yellowlees P, Burke Parish M, González Á, et al. Asynchronous telepsychiatry: A component of stepped integrated care. Telemed J E Health 2018; $24: 375-378$.
22. Kroll DS, Chakravartti A, Gasparrini K, et al. The walk-in clinic model improves access to psychiatry in primary care. J Psychosom Res 2016;89:11-15.

23. Chariatte V, Berchtold A, Akré C, et al. Missed appointments in an outpatient clinic for adolescents, an approach to predict the risk of missing. J Adolesc Health 2008;43:38-45.

24. Myles ZM, German RR, Wilson RJ, et al. Using a statistical process control chart during the quality assessment of cancer registry data. J Regist Manag 2011;38:162-165.

25. Lazare A. The psychiatric examination in the walk-in clinic. Hypothesis generation and hypothesis testing. Arch Gen Psychiatry 1976;33:96-102.

26. Lazare A, Eisenthal S, Wasserman L. The customer approach to patienthood. Attending to patient requests in a walk-in clinic. Arch Gen Psychiatry 1975; 32:553-558.

27. Younis MS, Al-Noaimi AS, Zaidan ZA, et al. Clinical and demographic profile of attendees at baghdad's walk-in psychiatric clinic. Oman Med J 2013;28: 365-370.

28. Dea RA. The integration of primary care and behavioral healthcare in northern California Kaiser-Permanente. Psychiatr Q 2000;71:17-29.

29. Robinson PJ, Strosahl KD. Behavioral health consultation and primary care: Lessons learned. J Clin Psychol Med Settings 2009;16:58-71.

30. Freeman DS, Manson L, Howard J, Hornberger J. Financing the Primary Care Behavioral Health Model. J Clin Psychol Med Settings 2018;25:197209.

31. Sunderji N, de Bibiana JT, Stergiopoulos V. Urgent psychiatric services: A scoping review. Can J Psychiatry 2015;60:393-402.

32. Kellermann AL. Crisis in the emergency department. N Engl J Med 2006;355:1300-1303.

33. Betz ME, Boudreaux ED. Managing suicidal patients in the emergency department. Ann Emerg Med 2016;67:276-282.

34. McManus MC, Cramer RJ, Boshier M, et al. Mental health and drivers of need in emergent and nonemergent emergency department (ED) use: Do living location and non-emergent care sources matter? Int J Environ Res Public Health 2018;15(1). 